\title{
Les premiers témoins imprimés de la prose d'“Ogier le Danois": dérimage ou réécriture?
}

\section{Aurélia Dompierre}

\section{(2) OpenEdition}

1 Journals

\section{Édition électronique}

URL : http://journals.openedition.org/studifrancesi/14575

DOI : 10.4000/studifrancesi. 14575

ISSN : 2421-5856

Éditeur

Rosenberg \& Sellier

\section{Édition imprimée}

Date de publication : 1 décembre 2018

Pagination : 308-406

ISSN : 0039-2944

\section{Référence électronique}

Aurélia Dompierre, «Les premiers témoins imprimés de la prose d"Ogier le Danois": dérimage ou réécriture? », Studi Francesi [En ligne], 186 (LXII | III) | 2018, mis en ligne le 01 décembre 2019, consulté le 07 janvier 2021. URL : http://journals.openedition.org/studifrancesi/14575; DOI : https://doi.org/ 10.4000/studifrancesi. 14575

\section{(c) (i) (9)}

Studi Francesi è distribuita con Licenza Creative Commons Attribuzione - Non commerciale - Non opere derivate 4.0 Internazionale. 


\title{
Les premiers témoins imprimés de la prose d" "Ogier le Danois": dérimage ou réécriture?
}

\begin{abstract}
No manuscript of Ogier le Danois in prose has become known to us, except the first 3 printed editions. Our study is based on the first one, from Vingle's edition dating from 1496. The aim is to analyse the amount of rewriting made by the prose writer based on the source: the poem in alexandrines dating from the XIV ${ }^{\text {th }}$ century. The question is whether the writer only removed the rhymes from the versified model or he proceeded to its rewording. Thus we will first study the changes of the form, and then the transformations of the contents. At last, we will analyse four thematics (feat of arms, representations of royal power, fantasy, hero) in order to understand whether the prose writer has renewed the epic subject or if he is in the continuity of his model.
\end{abstract}

La version française en prose de la légende d'Ogier le Danois $(P R)$ est le remaniement d'une version en alexandrins $(A L)$ encore inédite du milieu du XIV siècle, qui elle-même se fonde sur une version en décasyllabes datée des environs de 1310 qui ajoute une longue continuation à la Chevalerie Ogier, composée quant à elle aux alentours de $1200^{1}$. Aucun manuscrit de cette prose ne nous est parvenu. Nous en conservons néanmoins des exemplaires des trois premières éditions: celle de Jean de Vingle, exécutée à Lyon, dont il subsiste deux imprimés (Florence, Copenhague); celle pour Antoine Vérard, exécutée à Paris, dont il reste six exemplaires (Paris, Chantilly, Londres, Turin, Cologne, New York); celle enfin du Petit Laurens, réalisée à Paris, dont n'est conservé qu'un seul exemplaire (Paris, Arsenal)².

Nous nous proposons d'analyser ici la part de travail du translateur: s'est-il contenté de dérimer son modèle versifié ou bien a-t-il procédé à sa réécriture? Pour mener à bien cette enquête, nous avons confronté l'édition princeps de la prose par Jean de Vingle (6 octobre 1496), dans l'exemplaire, complet, conservé à Florence (BN, Palat. E-6-2-9), et la version en alexandrins telle qu'elle figure dans le ms. Arsenal 2985 (numérisé par Gallica), qui est le plus ancien témoin de $A L\left(2^{\mathrm{e}}\right.$ moitié du XIV ${ }^{\mathrm{e}}$ siècle selon la base JONAS de l'IRHT).

Dans cette étude, nous traiterons tout d'abord des remaniements de la forme, et envisagerons successivement les mutations structurelles, narratives et stylistiques. Ensuite, nous exposerons les remaniements saillants du contenu du texte-source. Enfin, nous procèderons à l'examen de quatre thématiques majeures - les faits d'armes, les figures du pouvoir royal, le merveilleux et le héros - afin de voir si le translateur renouvelle la matière épique ou bien s'il s’inscrit dans la continuité de son modèle.

(1) Cf. M. Отт, Ogier le Danois, Nouveau répertoire de mises en prose, (XIV'-XVI siècle), sous la dir. de M. Colombo Timelli, B. Ferrari, A. Schoysman et F. Suard, Paris, Classiques Garnier, 2014, pp. 623-642.

(2) Dans notre thèse (éd. de la prose d'Ogier le Danois dans les premiers imprimés), nous avons constaté que les trois premiers témoins sont extrêmement proches les uns des autres, qu'il s'agisse de la structure d'ensemble (Le Petit Laurens ajoute une rubrique en tête des $\$ 27,33$ et 518 ; il scinde ainsi en deux les chapitres VII, VIII et LVII, et fragmente donc le récit en 59 chapitres, et non en 57 comme Vingle et Vérard) ou du contenu, jusque dans le plus petit détail. 


\section{Remaniements de la forme}

Le passage du vers à la prose a donné lieu à diverses mutations formelles, touchant à la fois la structure, la technique narrative et le style.

\section{Mutations structurelles}

La réécriture en prose s'accompagne tout d'abord d'une réorganisation complète du récit-source, et celle-ci s'observe en premier lieu au niveau de la composition générale de l'œuvre.

Comme beaucoup de mises en prose du Xve siècle, Ogier le Danois résulte d'un abrègement considérable de son modèle. En effet, la matière très dense de $A L$, s'étendant sur 712 pages (soit environ 25000 vers), est réduite dans $P R$ à 314 pages. Le passage du vers à la prose se réalise donc par la perte de plus de la moitié de la masse textuelle.

De fait, si le prosateur conserve la composition générale de l'œuvre en cinq parties, il ne leur fait pas subir le même allègement et modifie leurs proportions respectives. Ainsi, les parties consacrées aux Enfances et à la Chevalerie, qui occupaient respectivement 126 et 264 pages dans $A L$, passent à 60 et 90 pages dans $P R$. Le déséquilibre initial entre ces deux parties est alors atténué au prix d'une réduction importante de la deuxième. Les trois parties suivantes, correspondant à la suite inventée par la continuation en décasyllabes, subissent elles aussi des réductions importantes, mais moindres: elles occupent désormais 164 pages contre les 319 de $A L$. Dans le détail, les aventures en Orient passent de 241 pages à 110, le séjour en Avalon de 31 à 17 et le retour d'Ogier en France après deux cents ans passés en Faerie de 47 à 36 . Le translateur a ainsi réduit le déséquilibre entre les deux premières parties et la suite, mais en procédant à une inversion: désormais, les parties ajoutées (164 pages) bénéficient de plus de place que les deux parties primitives (150 pages); et parmi elles, ce sont les aventures plus proprement merveilleuses et romanesques (parties IV et V) qui ont été les moins abrégées. Ces variations dans les proportions des parties favorisent alors un rééquilibrage de l'ensemble, et se révèlent aussi significatives des nouvelles orientations de l'œuvre.

Le translateur a également modifié les délimitations des quatre premières parties. Prenons tout d'abord le passage de la première à la deuxième: dans $A L$, la première partie prenait fin avec le départ de Caraheu, de Glorïande et de ses hommes, et le retour en France de Charlemagne et de ses seigneurs, après le rétablissement du siège papal, tandis que la deuxième commençait par l'arrivée des nouvelles de Bellicenne annonçant à Ogier l'existence de leur fils, suivie du récit des malheurs de son père, Gauffroy de Danemark, réfugié à Mayence à la suite de l'attaque du roi païen Mandaquin. Dans PR, en revanche, la formule de transition intervient à la suite du retour d'Ogier de son expédition au Danemark. Le prosateur a ainsi déplacé dans la partie consacrée aux Enfances un épisode qui ne relevait pas encore de la rébellion d'Ogier.

Pour ce qui est du passage de la deuxième à la troisième partie, dans $A L$, la transition était insérée après la séparation d'Ogier et de Caraheu, et avant la victoire des chrétiens sur les païens, le mariage d'Ogier avec la princesse Clarice et leur départ pour l'Angleterre. Cette délimitation mettait en évidence la scène majeure de la séparation des deux compagnons d'armes et créait ainsi un effet de symétrie avec la fin de la première partie, mais elle intervenait de façon surprenante en plein milieu d'un épisode et avant le début de la continuation. $P R$ modifie ce découpage en faisant commencer la troisième partie après les résolutions de l'intrigue de la Chevalerie, c'est-à-dire après l'embarquement du héros pour l'Angleterre.

Le passage de la troisième à la quatrième partie était abrupt dans $A L$ : la formule de liaison apparaissait en plein milieu du récit de la tempête en mer et l'évocation de l'arri- 
vée d'Ogier en Faerie. Aussi PR déplace-t-il les scènes de résolution (le mariage de Gautier et de Clarice, la condamnation des traîtres et le couronnement de Gautier devenu roi de Babylone) en clôture de la troisième partie, et la quatrième partie s'amorce alors avec le départ de Caraheu et d'Ogier en Inde. Cette nouvelle structure a pour intérêt de créer un effet de symétrie avec la fin de la deuxième partie puisque le mariage de Gautier et de Clarice fait écho à celui de son oncle avec Clarice d'Angleterre.

Aucune formule n'apparaît dans $A L$ pour assurer la transition entre la quatrième et la cinquième partie. Dans $P R$, c'est la rubrique d'un nouveau chapitre qui, seule, remplit cette fonction. Le translateur a en outre réinvesti le procédé de mise en abyme inventé par l'auteur de $A L$ : à son retour en France, après deux cents ans d'absence, Ogier rencontre un écuyer aux abords de Montpellier qui lui résume le roman qu'un de ses parents a fait de sa vie jusqu'à sa disparition en mer. Cette analepse, très développée, permet de relier la cinquième partie avec les trois premières.

En somme, l'auteur de $P R$ a conservé la composition générale de l'œuvre en cinq parties, mais il les a rééquilibrées tout en les abrégeant, et a repensé leurs délimitations, pour renforcer la cohérence de l'ensemble.

Le translateur opère également des mutations structurelles aux extrémités du récit. Le prologue de $P R$ se distingue en effet de son modèle par sa composition. Tout d'abord, alors que celui de $A L$ était étendu sur plus de 40 vers et débordait sur la deuxième laisse, celui de $P R$ est circonscrit à un seul paragraphe d'une longueur de 63 lignes. De plus, si le prologue de $A L$ ne faisait mention que de la Chevalerie (et non des Enfances) et annonçait les aventures féériques sur l'île d'Avalon, $P R$ résume les deux parties primitives de la chanson, mais passe sous silence les aventures nouvelles de la continuation. Ainsi, dans cette vitrine que constitue le seuil du livre s'affiche d'emblée le primat accordé aux parties originelles sur l'œuvre toute entière. Enfin, tandis que l'épisode de la venue des fées-marraines est raconté dans la troisième laisse de $A L$ et fait donc partie intégrante du récit, dans $P R$, il apparaît dans le Prologue. L'épisode est de cette manière situé hors de l'histoire, renvoyé dans une sorte de pré-histoire. Or, ce déplacement fait sens, puisque cette scène initiale programme toute la suite de l'œuvre.

Les deux versions ne proposent pas non plus la même fin. Dans $A L$, l'explicit remplit une fonction de clôture (avec l'emploi du passé simple dans Ainsi regna Ogier que Jhesucrist amoit). À l'inverse, la fin de PR est ouverte: la mort d'Ogier est mise en suspens puisque le tison n'est toujours pas consumé, comme le montre l'emploi du présent dans Mais, veu le tizon qui est encores en l'abbä̈e de Saint Pharon de Meaulx, bien enbarré de fer, [...] toute personne peut presumer sans difficulté qu'il est encores en vie. Ainsi, le héros danois vivrait encore, auprès de Morgue en Avalon, ou ailleurs. Par $P R$, Ogier atteint donc la dimension d'un héros mythique. La fin de l'œuvre racontant l'enlèvement d'Ogier par Morgue crée en outre un effet de boucle avec le début. Aussi, comme la fin est suspensive, le lecteur est-il libre d'imaginer qu'après un nouveau séjour en Avalon, Ogier reviendra en France pour sauver la chrétienté avant d'être à nouveau ravi par Morgue. L'œuvre reste ainsi inachevée, toujours en devenir ${ }^{3}$. Cette structure circulaire pourrait, nous semble-t-il, être interprétée comme une métaphore des réécritures successives de la légende d'Ogier le Danois.

(3) Cela fait écho au début de la $5^{\mathrm{e}}$ partie où Ogier refuse de voir son roman biographique terminé et exprime son désir de le continuer par l'accomplissement de nouveaux exploits: je le [= le rommant] feray donc croistre, car j'ay intencion de faire encores des prouesses qui n'y sont pas! (470/27-28). 
L'autre mutation structurelle de taille opérée par l'auteur de $P R$ relève de la création du double système de fragmentation que représentent la mise en paragraphes et la mise en chapitres.

$A L$ compte environ 24780 vers pour 675 laisses, soit des laisses de 36 vers en moyenne, c'est-à-dire plutôt brèves. De fait, si leur longueur est très variable (de 11 à 110 vers), seulement 2 dépassent les 100 vers. Cette version n'adopte donc pas un rythme romanesque. $P R$ non plus: sa matière constituée d'environ 11345 lignes est répartie en 527 paragraphes dont la longueur moyenne avoisine les 21,5 lignes (sur une échelle de 6 à 86 lignes). Plus de la moitié des paragraphes a un nombre de lignes égal ou inférieur à 20 , et les trois quarts n'excèdent pas 30 lignes. De là, deux constats: d'une part, $P R$ se caractérise par la prédominance des paragraphes courts, d'autre part, son texte est plus fragmenté que celui de son modèle. Le translateur a donc procédé à une restructuration de la matière.

Mais s'est-il pour cela servi de l'agencement des laisses comme modèle ou bien s'en est-il écarté pour proposer une nouvelle organisation? Il y a bien quelques passages où il a repris avec fidélité le découpage de sa source ${ }^{4}$. Mais, le plus souvent, il s'émancipe et procède au «démantèlement des laisses»". Sur quels critères s'estil alors appuyé? Il ressort de notre analyse que ce découpage sert souvent un but de dramatisation et de suspension. Il est notamment significatif que le changement de paragraphe puisse suspendre une discussion, une réponse décisive, plus souvent encore une réplique. Cette mise en attente est rendue encore plus flagrante par des découpages particulièrement surprenants, comme lorsqu'une phrase est interrompue et laisse en suspens la réaction d'un personnage; ou quand une phrase au discours direct est scindée en deux; ou quand une proposition introductrice de discours direct est séparée des paroles rapportées; ou encore lorsque le verbum dicendi est séparé des paroles par le changement de paragraphe et le changement de chapitre; ou enfin quand une proposition circonstancielle est séparée de sa principale. La recherche constante de cet effet de suspension pourrait, nous semble-t-il, s'interpréter comme un vestige du lyrisme de la Chevalerie Ogier de 1200.

Le travail de structuration mis en œuvre par l'auteur de $P R$ s'observe par ailleurs dans sa création de chapitres. Le texte est réparti en 57 chapitres de taille variable: le plus bref - le chap. IV - n'occupe qu'une page (24 lignes); le plus étendu - le chap. LIV - en couvre 13 (environ 507 lignes). Au-delà de ces cas extrêmes, leur longueur moyenne est de 5 pages et demi (235 lignes environ). Les chapitres les plus longs se situent dans les parties ajoutées par la continuation, et particulièrement les troisième et cinquième parties.

Ces variations dans l'étendue des chapitres nous renseignent entre autres sur l'attitude du translateur face aux sujets que lui propose son modèle. Ainsi, significativement, les chapitres les plus courts ne sont jamais consacrés aux faits d'armes d'Ogier; ils servent à raconter des scènes transitoires ${ }^{6}$, ou des scènes préparatoires ${ }^{7}$ ou

(4) Voir l'ex. donné par E. Poulain-Gautret, concernant le retour d'Ogier en France après deux cents ans (La Tradition littéraire d'Ogier le Danois après le XIII siècle. Permanence et renouvellement du genre épique médiéval, Paris, Champion, 2005, pp. 60-61).

(5) Ibid., p. 62.

(6) Par ex., le chap. IV, qui traite de la rencontre de Charlemagne avec le pape et de l'installation de son armée à Suze, se situe entre le récit du voyage pour l'Italie et celui de la bataille contre les païens ayant pris Rome.

(7) Par ex., le chap. XXV raconte la décision de Bruhier d'aller en France et la préparation de son armée. 
encore des scènes de résolution ${ }^{8}$. Quant aux chapitres les plus longs, ils juxtaposent différentes péripéties, souvent marquées par des rebondissements; ils regroupent des scènes d'action et des épisodes romanesques, comme le séjour d'Ogier en Avalon.

Quels sont les critères qui ont déterminé la délimitation des chapitres? Le prosateur ne s'est pas contenté de la facilité qui aurait consisté à s'appuyer sur les formules stéréotypées de transition présentes dans son modèle. Les débuts de chapitres correspondent rarement, en effet, au début des laisses. Pour la seule partie consacrée aux Enfances, nous n'avons relevé qu'un cas de coïncidence exacte (le chap. VII). Les ouvertures des autres chapitres sont en décalage avec celles de AL: elles correspondent à ce qui est raconté au milieu ou à la fin d'une laisse. Ce ne sont donc pas les repères donnés par la source qui ont servi au découpage en chapitres. Leur structuration n'obéit pas non plus à des principes rigides. Un chapitre peut en effet avoir pour fonction d'isoler un épisode dans son intégralité, mais un même épisode peut aussi être réparti en deux chapitres ou plus. À l'inverse, un chapitre peut regrouper des épisodes distincts. Le changement de chapitre peut en outre isoler certaines étapes de la narration: un élément perturbateur, un retournement de situation ou un dénouement. Il peut enfin coïncider avec un changement de personnage. L'usage est donc fluctuant. Toutefois, il se dessine des constantes, notamment le goût pour les fins suspensives. Les clôtures de chapitres sont souvent, en effet, les préludes de grandes scènes épiques développées dans le chapitre suivant; les longs chapitres s'arrêtent même régulièrement juste avant une scène de combat. Les fins de chapitres correspondent fréquemment aussi à des moments de forte tension dramatique, où le sort des personnages est en suspens; le changement de chapitre peut même alors apparaître comme l'expression matérielle de l'attente des protagonistes'. La fréquence des scènes de départ en fin de chapitre crée également un effet d'attente ${ }^{10}$.

La mise en suspens de l'action semble donc bien, par sa fréquence, avoir été un critère fondamental pour le découpage de la matière en chapitres. Ceux-ci ne constituent pas des unités closes et il s'en dégage moins un effet de rupture qu'un effet de continuité. Aussi le chapitre IV, qui se réduit à un seul paragraphe et qui fonctionne comme une transition, est-il représentatif de cette technique narrative, voire de l'œuvre tout entière dont la fin est ouverte.

L'analyse des ouvertures de chapitres corrobore d'ailleurs ce constat. Plusieurs procédés linguistiques et narratifs renforcent en effet le lien entre les clôtures et les ouvertures de chapitres, comme des termes anaphoriques, ou la reprise de segments clôturant le chapitre précédent, ou enfin des interventions du narrateur, placées en clôture ou en ouverture. Le translateur a donc veillé à garder le fil du récit, malgré l'interruption momentanée occasionnée par les rubriques.

La formulation de ces titres de chapitres se limite à quelques moules et clichés, avec l'emploi récurrent de la structure verbale introduite par Comment/ Com $(m) e$... Cette stéréotypie est aussi mise en œuvre par tout un jeu de répétitions: entre les rubriques et le texte, mais aussi entre les rubriques elles-mêmes. Certaines se retrouvent, en effet, reliées les unes aux autres par la reprise de formules similaires qui se font écho. Deux thèmes reviennent de façon récurrente: la bataille et la navi-

(8) Par ex., le chap. XXXI relate le mariage d'Ogier et de Clarice, puis leur départ pour Paris.

(9) Cf. chap. I (Ogier dans l'attente de la réponse de son père dont sa vie dépend) et XVI (attente de l'armée de Charlemagne par celle de Desier).

(10) Le dialogue à cheval sur les chapitres XXXV-XXXVI participe du même but. 
gation en mer. Par le biais des rubriques se dessinent donc des leitmotive. D'ailleurs, leur analyse lexicale met en évidence les sujets de prédilection du translateur: les thématiques du déplacement ${ }^{11}$ et de la guerre ${ }^{12}$. En ce sens, les titres laissent bien apparaître «une hiérarchie des signifiés» ${ }^{13}$.

Quelles sont alors les fonctions assignées à ces rubriques? Elles offrent tout d'abord des repères visuels au lecteur et participent de la structuration de la matière. Elles assument également une valeur informative puisqu'elles résument et annoncent le contenu du chapitre à venir. Mais les rapports qu'elles entretiennent avec le chapitre sont très variables.

Douze titres offrent des résumés parfaitement exhaustifs. Plusieurs révèlent même d'emblée l'élément de résolution des péripéties. Ces rubriques n'ont donc pas pour rôle de créer un effet de suspense chez le lecteur qui est au contraire placé ici en position d'omniscience.

Le résumé fourni par 39 autres rubriques est, en revanche, incomplet; leurs lacunes sont parfois même béantes. La rubrique du premier chapitre est exemplaire et programmatique à ce sujet: elle se contente de reformuler le début de ce qui suit (Comment le duc manda tous ses parens et amys pour faire l'obseque de la dame et pour baptiser son filz, lequel fut nommé Ogier), mais elle passe sous silence les actions de premier plan racontées ultérieurement, à savoir le second mariage du duc, la naissance de son second fils Guyon, la menace de Charlemagne qui envoie un messager auprès du duc, le refus d'obtempérer de celui-ci, la première guerre opposant les armées française et danoise, les accords fixés, la promesse mensongère du duc, la captivité d'Ogier chez le châtelain Garnier de Saint-Omer et enfin l'envoi par l'empereur de quatre ambassadeurs au Danemark. Les lacunes d'autres titres portent entre autres sur le personnage de Caraheu et sa conversion, ainsi que sur les aventures d'Ogier en Faerie. La rubrique n'a donc pas toujours pour fonction de mettre en valeur des éléments importants du chapitre. Les tris opérés sur le récit qui la suit lui confèrent plutôt une valeur interprétative, et peut-être aussi une valeur publicitaire, dans le sens où elle constituerait une sorte de vitrine du livre mettant en avant des sujets épiques.

Restent enfin 5 rubriques qui ne coïncident pas avec le contenu du chapitre. Trois d'entre elles sont en avance sur la narration. Ainsi, la rubrique du chap. XXIV annonce la délivrance d'Ogier pour combattre Bruhier alors que le chapitre se clôt sur l'emprisonnement du héros. De même, celles des chap. XLVII et LII anticipent sur des événements racontés dans les chapitres qui suivent dont les rubriques deviennent alors redondantes. Ces annonces à longue distance participent encore de cette recherche de mise en suspens déjà maintes fois constatée.

Deux faits sont donc à retenir sur les mutations structurelles opérées par le translateur: premièrement, $P R$ offre une nouvelle composition qui fait sens et qui contribue à renforcer la cohérence de l'ensemble; deuxièmement, la fragmentation du texte en paragraphes et chapitres est nettement marquée par le goût pour l'effet de suspension et de continuité, et pour la dramatisation.

(11) Sur 57 rubriques, 39 annoncent un départ et/ou une arrivée de personnages.

(12) 14 annoncent un duel, 17 une bataille et 6 le siège ou l'assaut d'une place forte. L'examen des illustrations de l'édition de Vingle témoigne également d'une forte récurrence des scènes de combat $(57 \%)$ et de voyages en mer (19\%) (avec réemploi des mêmes bois).

(13) P. Demarolle, De la narratologie à la syntaxe: les titres des chapitres de la mise en prose de "Garin le Lorrain" par Philippe de Vigneulle, dans Rbétorique et mise en prose au XV siècle. Actes du VI colloque international sur le moyen français (Milan, 4-6 mai 1988), t. II, Milano, Vita e Pensiero, 1991, p. 247. 


\section{Mutations narratives}

Le passage du vers à la prose se traduit aussi par des mutations narratives, perceptibles d'une part dans le traitement des interventions du narrateur, d'autre part dans celui des bouleversements chronologiques.

Concernant tout d'abord les interventions du narrateur, dans $A L$, elles assumaient essentiellement deux fonctions: elles contribuaient à la structuration du récit, en ouvrant les laisses ou en assurant les transitions, et surtout elles servaient de chevilles. Le passage à la prose a permis au texte de se libérer de ces contraintes du vers, de ces formules artificielles et hiératisantes. $P R$ ne compte plus que 231 occurrences de je narratoriel.

Comme dans son modèle, les interventions explicites du narrateur ont d'abord une fonction structurante (près de $37,5 \%$ ). Elles assurent alors soit des transitions, soit des renvois, soit des anticipations. Dans tous les cas, leur formulation est répétitive et stéréotypée. Mais, si elles contiennent de façon symptomatique des verbes de parole (parler, taire, ouyr), mimant l'oralité des chansons de geste, ces marques d'oralité sont souvent aussi associées à des adverbes qui font référence au cotexte, tels que (i)cy, dessus, devant, desja. Cette opposition rend bien compte du statut ambivalent du mode de réception du livre, destiné à la fois aux escoutans et lisans (Prologue/5). Quoi qu'il en soit, dans $P R$, la fonction de régie est donc bien toujours prise en charge par le narrateur; ses interventions organisatrices n'innovent donc pas.

Comme celui de $A L$, le narrateur de $P R$ fait aussi des interventions métanarratives pour commenter son art de raconter (un peu plus de $11 \%$ ). De fait, conformément à la tradition de la chanson de geste, il souligne à plusieurs reprises la véracité de l'histoire qu'il raconte; il se réfère aussi à des sources présumées qui prennent des appellations variées: il s'agit tantôt du conte, tantôt de l'istoire, tantôt de la (ou des) cronique(s). Ainsi, le narrateur de PR se présente toujours comme un simple transmetteur. Enfin, plusieurs de ses interventions métanarratives indiquent son souci de raccourcir la matière et d'éviter les digressions. Le translateur n'innove donc pas non plus à ce niveau.

En revanche, $P R$ se démarque de son modèle par son emploi fréquent d'interventions participatives, rares dans $A L$. Nous en avons comptabilisé 118 dans $P R$ (un peu plus de $51 \%$ ); 37 d'entre elles visent à faire participer les destinataires du récit et recourent à différents procédés rhétoriques favorisant leur captatio, notamment les formules présentatives stéréotypées introduites par Qui veist, Qui eust veu et Si veissiez. Le translateur réinvestit ainsi les clichés de la rhétorique de la chanson de geste, abandonnées dans son modèle; il a en revanche fait disparaître les formules stéréotypées du type Seigneurs oyés chançon ou Seigneurs or entendés, présentes ici et là dans $A L$. Dans les 81 interventions participatives restantes, le narrateur manifeste sa propre implication au récit, et c'est là l'une des originalités de $P R$. Tantôt il exprime ses émotions ou pensées à la p1; tantôt il s'investit dans le récit tout en impliquant ses destinataires avec la p4; tantôt enfin, sous des dehors plus objectifs, il formule des jugements d'approbation ou de désapprobation sur les actions qu'il raconte, jugements étayés parfois par des expressions proverbiales.

L'auteur de $P R$ a donc diversifié les interventions du narrateur. Il a non seulement repris les clichés et stéréotypes de la chanson de geste, mais a su rendre son œuvre plus personnelle à travers notamment l'insertion de commentaires participatifs.

Pour ce qui est à présent de l'ordre de la narration, dans $P R$, la linéarité de la narration est encore régulièrement interrompue par des bouleversements chronologiques. 
Les prolepses restent assez nombreuses, malgré une nette réduction de celles qui sont prises en charge par le narrateur. $A L$ affichait, en effet, un goût prononcé pour ce procédé, souvent utilisé à la fin des laisses. Dans $P R$, l'emploi des anticipations énoncées par le narrateur devient plus espacé et s'applique davantage à des événements de premier plan. Mais cette perte est compensée par la présence des rubriques qui annoncent le contenu du chapitre à venir. Le translateur a en revanche conservé la plupart des anticipations prises en charge par les personnages. Ainsi, l'œuvre s'ouvre toujours sur la scène programmatique inventée par l'auteur de $A L$, où les fées-marraines scellent par leurs dons le destin du héros et dessinent une ligne d'attente pour les lecteurs. Les personnages continuent aussi de recevoir des visites d'anges ou de faire des rêves prémonitoires, ou enfin prononcent de discrets commentaires à valeur proleptique.

Quant aux analepses, là encore la part de celles qui sont prises en charge par le narrateur est moindre. Plus fréquentes sont celles qui sont prises en charge par les personnages, et c'est en premier lieu à Ogier que revient ce rôle. Comme dans $A L$, en effet, le héros de $P R$ se trouve très souvent en situation de se raconter. Le récit de ses infortunes constitue d'ailleurs un leitmotiv de l'œuvre, à travers la reprise sans cesse déclinée du motif du planctus. Celui-ci apparait dès la partie consacrée aux Enfances à l'occasion de l'affaire du chevage qui place le jeune otage en position de victime sacrificielle. Le planctus réapparaît ensuite dans la partie consacrée à la Chevalerie, à la suite du meurtre de Baudouin, et il prend de l'ampleur au fil des pages. Plus tard, dans l'épisode de Châteaufort, c'est la perte de ses compagnons, sortes de fils de substitution, qui occasionne une nouvelle scène de lamentation où Ogier récapitule toutes ses infortunes. Le motif du planctus est enfin réinvesti dans l'épisode en Orient; cette fois, ce sont les différentes trahisons subies par Ogier qui sont le moteur de ses plaintes. Le héros est du reste relayé dans le récit de ses malheurs par d'autres personnages. Ce leitmotiv cumule plusieurs fonctions: structurelle, puisqu'il permet de récapituler les faits antérieurs; lyrique et pathétique, du fait qu'il exprime les souffrances d'Ogier; tragique, parce que le héros semble voué à répéter éternellement ses plaintes et à ressasser ses peines; parfois aussi dramatique, parce que ses monologues sont souvent entendus par des espies et la révélation de leur contenu entraîne des retournements de situation. Ogier se trouve par ailleurs fréquemment dans la position de conteur, voire de fabulateur. Dans la dernière partie notamment, de retour en France après deux cents ans d'absence, il est à plusieurs reprises amené à faire le récit de ses malheurs et gloires passées à l'époque révolue de Charlemagne: à son aubergiste de Paris (479/29-32), au roi Philippe de France (514/20-32) et enfin à la reine de France (521/4-15). Ses actions passées sont en outre racontées par les autres personnages. À la manière d'un kaléidoscope, le récit des actions d'Ogier se réfracte donc en une multitude de petits récits enchâssés. Cette démultiplication des récits est menée à son comble par la reprise, que nous avons déjà signalée, de la mise en abyme inventée par l'auteur de $A L$, où Ogier, après deux cents ans passés en Faerie, revient en France et rencontre un écuyer qui lui fait un long résumé du beau livre en rommant sur Ogier le Danois (469/17). D'autres personnages procèdent à des retours en arrière: Gautier, double d'Ogier, est le second protagoniste amené à souvent conter des actions passées. Plusieurs motifs rhétoriques repris du modèle donnent aussi lieu à des retours en arrière: ce sont les conseils de barons, les lettres, les messages, les anges, les songes.

En somme, $P R$ recourt plus aux analepses qu'aux prolepses. Est-ce à dire que l'œuvre est plus tournée vers le passé que vers l'avenir et qu'elle se soucie plus de «re- 
produire que de recréer» ${ }^{14}$ ? Peut-être en ce qui concerne le fond, moins assurément pour ce qui est de la forme. En tout cas, leur nombre encore important contribue non seulement à la cohérence de l'ensemble, mais témoigne aussi et surtout d'une persistance du «'plaisir régressif' de la répétition» ${ }^{15}$ propre à la chanson de geste.

\section{Mutations stylistiques}

Le passage du vers à la prose a enfin donné lieu à des mutations stylistiques.

Le translateur a tout d'abord gommé des traits récurrents et caractéristiques de AL. PR est ainsi marqué par l'abandon des formules-chevilles, entraînant la disparition de la plupart des épithètes de nature, des interventions du narrateur faisant référence à la source du roman, et des figures de style, notamment des métaphores; la suppression de la plupart des toponymes; l'élagage des noms propres des personnages, avec une tendance à l'anonymisation; la réduction voire l'élimination des listes de noms propres au profit d'expressions englobantes.

A contrario, le passage à la prose se caractérise par le développement d'autres traits stylistiques, dont les plus marquants sont la forme «diffuse» de la phrase ${ }^{16}$ et le recours fréquent à l'amplificatio. Le prosateur a en effet accentué l'allongement de la phrase déjà amorcé par l'auteur de $A L$. Il a pour cela fait jouer les constructions synthétiques (avec l'emploi de propositions infinitives, de constructions participiales, ou du relatif de liaison de transition inséré dans une construction absolue du type Lesquelles parolles dictes...) et les constructions analytiques (avec le recours abondant de subordonnées et de compléments s'enchaînant les uns les autres), au risque de certaines lourdeurs, voire de ruptures de construction. $P R$ se caractérise également par l'usage régulier de l'amplificatio. Ainsi, il ne rechigne pas devant les répétitions ${ }^{17}$ ni les pléonasmes ${ }^{18}$ et les «figures étymologiques» ${ }^{19}$. Fait plus marquant encore, il exploite abondamment les itérations (pseudo-)synonymiques, caractéristiques en particulier de la prose narrative du $\mathrm{Xv}^{\mathrm{e}}$ siècle. Ainsi, les séries binaires et ternaires, pour la plupart du fait du prosateur, parsèment le texte; elles peuvent alors être redoublées ${ }^{20}$; un trinôme synonymique peut même être relayé par un binôme ${ }^{21}$, ou l'inverse ${ }^{22}$. Comme

(14) E. Poulain-Gautret, La Tradition littéraire d'Ogier le Danois après le XIII' siècle cit., p. 79.

(15) Ibid., p. 80.

(16) Expression empruntée à J. RASMUSSEN, La Prose narrative française du XV siècle. Étude esthétique et stylistique, København, Munksgaard, 1958, p. 42.

(17) Par ex.: celle maudite gens, mauldictz Sarrazins et infideles (10/9), la belle Gloriande [...] vestue d'ung bliant sarrasinoys, auquel bliant une payenne avoit esté neuf ans a le faire-et ledit bliant estoit noblement et tres richement garny de moult riche pierrerie et tout semé de belles perles orientalles, si que le pareil bliant jamais homme qui fut vivant sur terre n'avoit veu (23/62-66).

(18) Par ex.: les Sarrazins reculez arriere (18/1), descendre en bas (152/23).

(19) Expression inventée par L. FLEURIOT pour désigner un redoublement du verbe dont la racine se retrouve dans son propre complément (Notes lexicographiques et philologiques (langues celtiques), rééd. d'articles parus dans les «Études Celtiques» avec un index général établi par G. Le Menn, Skol, 1997, pp. 46-47). Formule reprise notamment par M. Colombo Timelli, (éd.) L' "Histoire d'Erec en prose”. Un roman du XV siècle, Genève, Droz, 2000, p. 86. Par ex.: je te donne ung don (1/49), de ses armes se fist armer (15/48), le songe que il avoit songé (26/6-7), c'est ung messager faint qui faignant venir... (30/12).

(20) Par ex.: le venir servir et luy rendre la feaulté et hommage (5/29), une chose bideuse et tres abbominable de les regarder, et chose trop inbumaine (5/48-49), le parjurement et oultrage de vostre orguilleux et depiteux pere (8/30-31).

(21) Par ex.: la plus belle, la plus honnorable et la plus parfaicte en toute beauté et vertu (23/50-51), la vie mauvaise, parverse et douloureuse du maudit et faulx traytre Brunamon (67/47-48).

(22) Par ex.: Or est nostre joyeuse esperance et attente tournee sur nous, et tres douleureuse et tres vituperable perte, confusion et grant deshonneur (409/19-21). 
l'indique Fr. Suard, ces séries coordonnées n'ont pas qu'une fonction ornementale: elles «concourent à l'amplification rythmique plus qu'à l'intensification du sens» ${ }^{23}$.

En définitive, l'auteur de $P R$ a accompli un véritable travail de remise en forme de son modèle, tant structurelle que narrative et stylistique. Nous allons voir à présent que la réécriture est également marquée par des modifications relatives au contenu de l'œuvre.

\section{Remaniements du contenu}

Le translateur a remanié la matière de sa source en procédant tantôt à des réductions voire à des suppressions, tantôt à des amplifications voire à des ajouts.

1. Suppression et réduction significatives de motifs, de scènes, voire d'épisodes entiers

L'un des faits les plus marquants du passage à la prose est la réduction systématique des motifs narratifs guerriers. L'abrègement touche en premier lieu les scènes d'armement. Elles peuvent être supprimées, comme celles évoquant l'équipement d'Ogier et de ses hommes pour son expédition à Mayence, ou celui du héros fuyant le palais de Laon. Mais, le plus souvent, elles sont réduites à un ou des terme(s) résomptif(s) comme les verbes appareiller et mettre en point. La réduction la plus flagrante en la matière concerne le passage évoquant l'équipement de Caraheu. Il s'étendait sur 2 laisses, soit 37 vers, dans $A L ; P R$ n'y consacre plus que 10 lignes, dénuées de précisions. Le topos épique de l'armement des chevaliers a donc été délaissé par le translateur (et cela est sans doute à relier à son désintérêt pour les descriptions, évoqué ci-dessous). Le récit même des combats a également subi d'importantes réductions. Ainsi, les références au sang répandu, aux armes brisées et aux chevaux tués sont régulièrement éliminées. Quant aux différents coups portés, ils sont souvent eux aussi évoqués par un terme résomptif, comme dans firent la grant desconfiture des payens (42/2). La mort des ennemis est d'ailleurs elle aussi souvent vite expédiée. Le motif du combat n'est donc plus une source d'inspiration pour le translateur.

La réécriture en prose est également marquée par l'accentuation d'une tendance, déjà amorcée par $A L$ d'après les analyses d'E. Poulain-Gautret ${ }^{24}$, consistant à réduire la part des descriptions traditionnelles.

Pour ce qui est tout d'abord des décors, la part qui leur est désormais consacrée est minime. Plus aucune pause descriptive ne vient en effet agrémenter le récit des deux parties primitives. Ainsi, la longue description de Châteaufort, qui s'étendait dans $A L$ sur 30 vers environ, se réduit désormais à la seule mention de la grande (121/2) et baulte tour (121/4). Les trois parties de la continuation, plus dépaysantes et plus pittoresques, n'ont pas davantage inspiré notre auteur. Comme dans $A L$, aucune description dans la troisième partie n'exploite la couleur locale du cadre oriental. De même, dans la quatrième partie, la description de l'île d'Avalon et du château d'Aymant, qui occupait 15 vers dans $A L$, se limite désormais à une ligne: ung moult riche et triumphant chasteau tout fait de pierres precieuses (514/28-29). La description du lit merveilleux reste néanmoins développée sur 6 lignes; cette chose merveilleuse

(23) Guillaume d'Orange - Étude du roman en prose, Paris, Champion, 1979, p. 231.

(24) E. Poulain-Gautret, La Tradition littéraire d'Ogier le Danois après le XIII' siècle cit., pp. 101-102. 
est d'ailleurs le seul élément décoratif qui bénéficie encore d'une description à part entière dans $P R$.

La part accordée aux portraits se voit elle aussi fortement diminuée dans $P R$. Ils sont essentiellement situés dans les parties primitives. Le sort réservé aux héros épiques est à ce sujet tout à fait significatif. Tout d'abord, le translateur n'a pas conservé le portrait physique d'Ogier présent dans $A L$, en contrepartie, il a disséminé ici et là des bribes descriptives, toutes stéréotypées et hyperboliques. Le portrait de Gautier est de la même façon fragmenté et repose sur le même jeu de variété des points de vue et de répétitions. Quant au portrait physique de Caraheu, précis dans le modèle, il se réduit désormais à une accumulation de formules générales. Parmi les belles dames du rommant, la païenne Gloriande est la seule qui bénéficie encore d'un portrait physique développé (14 lignes contre 18 vers dans $A L$ ). Mais la conservation de cette pause descriptive traduit moins, nous semble-t-il, la volonté du translateur de favoriser le personnage - dont finalement il est dit peu de choses sur son corps, à l'exception de sa beauté et de ses cheveux longs et blonds - que son goût pour l'extraordinaire: le thème décrit ici, mis en relief par une anaphore, est bien avant tout la chose merveilleuse que constitue le bliaud confectionné par une fée. D'ailleurs, l'autre portrait de la jeune princesse en tenue d'apparat à l'occasion du duel d'Ogier et de Caraheu a disparu. Quant aux autres femmes du roman, elles seront moins décrites, voire pas du tout. Ainsi, Richemonde, l'épouse du roi de Pavie et l'amante secrète d'Ogier, ne bénéficie plus que d'un très bref portrait physique évoquant la beauté de son corps nu: elle estoit blanche et nette comme une belle perle (115/21-22). Quant à la reine de France, ce ne sont plus que ses actes et ses paroles qui la caractérisent. Enfin, le portrait de Morgue, l'amante officielle d'Ogier, reste tout aussi court que dans AL. Ainsi, ni les héros épiques, ni les femmes dont Ogier tombe amoureux ne suscitent de développement descriptif, ils sont au contraire l'objet de nouveaux abrègements. $P R$, en cela, se distingue du genre romanesque et se rapproche davantage de l'esthétique du conte.

Finalement, ce sont surtout les géants païens qui bénéficient encore de portraits physiques relativement étoffés - même s'ils se composent tous des mêmes traits stéréotypés, ceux qui sont traditionnellement appliqués aux païens dans les chansons de geste ${ }^{25}$. «C'est qu'effectivement la merveille est seule digne d'attirer le regard, et par là même, la description» ${ }^{26}$. D'ailleurs, soulignons-le, le seul portrait physique développé d'Ogier dans $P R$ est celui où on le voit dans trois scènes successives se transformer en un vieillard de deux cents ans après la perte de l'anneau de jeunesse. Dans $P R$, la description est donc réservée pour l'essentiel à la merveille.

$P R$ se caractérise aussi par l'abrègement, parfois très important, d'épisodes. L'exemple le plus marquant dans la partie consacrée aux Enfances est le second épisode lié à Mayence, comprenant l'évocation de la famine dans la ville, l'attaque des chrétiens contre les païens et la reconquête du Danemark par Ogier. Le translateur a supprimé la longue description exposant la situation contrastée des chrétiens soumis à la famine et des païens se régalant de divers mets; il a d'ailleurs aussi éliminé toutes les autres références à la nourriture; il a fait disparaître tous les passages dialogués; il a résumé l'affrontement des deux armées ainsi que l'expédition lancée par Ogier contre les païens pour reconquérir le Danemark; il a expédié la mort du duc en le fai-

(25) Cf. Ph. MÉNARD, Le Rire et le sourire dans le roman courtois en France et au Moyen Âge (1150-1250), Genève, Droz, 1969, pp. 47-52.

(26) E. Poulain-Gautret, La Tradition littéraire d'Ogier le Danois après le XiII siècle cit., p. 105. 
sant mourir sur le champ de bataille et, en supprimant le récit de son agonie auprès de ses proches, il a éliminé toute forme de pathos en faveur du traïtre; il n'a pas non plus mentionné la présence de la marastre d'Ogier et de son frère Guyon ni le bon accueil qu'Ogier a reçu à son arrivée par ceux-ci et les habitants de la ville, ni même enfin l'attribution du pouvoir à son frère. Si cet épisode a par ailleurs reçu des précisions sur l'organisation politique du Danemark, il a donc été fortement dégraissé; son récit n'est plus circonstancié, mais presque uniquement factuel.

Le passage à la prose se traduit en outre par la suppression de scènes plus ou moins longues. La première concerne l'arrivée d'un messager qui annonce la venue prochaine de Charlot, de retour d'Allemagne. De la laisse de $A L$ n'est finalement gardée que la décision de Charlemagne de lancer un assaut contre les Infidèles. Le translateur supprime également tout le contenu d'une laisse qui relatait le trajet d'Auquetin le Normand jusqu'à la tente de Charlemagne, puis son annonce du duel d'Ogier contre Brunamon; il élimine aussi une bonne partie de la laisse suivante, qui racontait la réaction de Charlemagne. Se dessine là une tendance de $P R$ : l'évacuation du récit des allées et venues des personnages secondaires. Les fêtes païennes sont du reste régulièrement passées sous silence, comme celle qui est organisée à l'annonce du mariage de Caraheu et Gloriande ou encore celle qui est menée par les païens à Rome, développée dans $A L$. Le translateur n'a pas tenu à exploiter la couleur locale qu'offre ce type de scène.

Le processus d'abrègement à l'œuvre dans $P R$ se manifeste enfin à travers la disparition de tout un épisode de la quatrième partie, qui mettait en scène Meurvin, le fils d'Ogier et de Morgue.

Dans $A L$, il prenait place alors que cela faisait déjà cent ans qu'Ogier séjournait en Faerie et que Meurvin avait atteint l'âge de trente ans. Morgue racontait alors à son amant la situation dramatique du royaume de France et de la chrétienté. Refusant de laisser partir Ogier, la fée lui expliquait que ce serait leur fils qui irait en France et elle lui annonçait les péripéties que le jeune homme connaîtrait avec son compagnon Drogues. Convaincu, Ogier donnait alors ses instructions à Meurvin: il lui conseillait de se rendre en France et de s'y présenter comme son fils, puis d'aller retrouver les fils de Gautier au Danemark. Aussitôt après Morgue transportait Meurvin en France, et il s'ensuivait un résumé de ses aventures. Le récit de cet épisode se clôturait par une intervention du narrateur exprimant son refus de poursuivre sa digression.

L'auteur de PR a choisi de faire l'ellipse de tout cet épisode excentré de l'histoire de son héros. Il s'est contenté de résumer les deux cents ans passés en Faerie avant d'évoquer les circonstances du retour d'Ogier en France. Par là, il a éliminé une incohérence: dans $A L$, Ogier mentionnait en effet l'existence des fils de Gautier à Meurvin (p. 656, v. 33) alors que le récit indiquait par ailleurs qu'il n'avait aucun souvenir de ses proches sous l'effet de la couronne d'oubli que lui avait fait porter Morgue. Cette suppression a donc ici une valeur de rectification.

\section{Ajouts et amplifications}

Parallèlement à toutes ces formes d'abrègement du récit-source, le translateur a opéré des ajouts et des amplifications.

Tout d'abord, il se complaît davantage que son prédécesseur à l'expression des sentiments et émotions des personnages. C'est avant tout le cas d'Ogier. Ainsi, dans la partie consacrée aux Enfances, le translateur insère plusieurs passages donnant accès à l'intériorité du jeune héros, alors otage de Charlemagne: il insère d'abord 
un discours mêlant prière et plainte suivi de l'évanouissement d'Ogier, puis une formule de transition en tête du paragraphe 5 qui exprime sur 4 lignes sa grant douleur. $P R$ développe également l'expression des sentiments de Charlemagne: par exemple, son ressenti à l'annonce de la capture d'Ogier par les païens, limité à un vers dans AL (Ainsi fu regretés li damoisiaux membrus, p. 78, v. 5), s'étend désormais sur 7 lignes. Ces inventions concourent à susciter l'émotion en faveur des héros chrétiens. Mais les personnages païens ne sont pas en reste. Le couple de Caraheu et de Gloriande semble même avoir particulièrement inspiré le translateur qui s'est plu à ajouter ou développer des passages évoquant leur intériorité. Ainsi, parmi de nombreux exemples, nous relevons le paragraphe 63, qui est une invention du prosateur, consacré exclusivement à l'expression des tourments de Glorïande, qui, accusée à tort de trahison, voit son sort suspendu à l'issue du duel entrepris par son champion Ogier contre Brunamon. L'autre exemple significatif est le paragraphe 350, autre création du translateur, consacré au portrait moral et psychologique de Caraheu: l'intervention du narrateur qui ouvre le paragraphe attire l'attention du lecteur sur l'extrême loyauté du roi païen, puis un monologue intérieur nous fait part des sentiments et des pensées de celui-ci. De telles amplifications traduisent l'intérêt du translateur pour ce couple de païens auquel il accorde des développements d'inspiration romanesque. $P R$ donne donc une place plus importante à l'intériorité des personnages que sa source - sans pour autant que l'on puisse parler de véritable analyse psychologique.

$P R$ se caractérise également par l'amplification des principaux épisodes amoureux de l'intrigue. Ainsi, le translateur développe l'idylle entre le jeune Ogier et Bellicenne. Il donne corps tout d'abord à la jeune amante en lui accordant un bref portrait, certes stéréotypé, mais qui a néanmoins le mérite d'exister (tant estoit plaine de beaulté, sens, maintien et noble contenement, 3/66). La relation amoureuse des deux jeunes gens prend ensuite une plus grande consistance avec l'ajout de précisions sur leurs premiers émois (laquelle Amours avoit ja ferue et attainte de son dard, qui tant luy getta d'amoureux et sourrians regars que force luy fut laisser le pleur et lamentacion, et convertir tout son dueil en l'amoureux deduit, 3/66-69) et les sentiments du jeune héros (la estoit amoureux de la plus mignonne dame que l'on savroit choisir ne regarder, et [...] nuyt ne jour ne pouoit reposer tant estoit feru de son amour, 9/11-13). De plus, dans $A L$, lorsque Bellicenne, au moment de leur séparation, annonçait à Ogier qu'elle était enceinte de lui, celui-ci ne s'engageait en rien auprès d'elle pour préserver son honneur et la protéger de la réaction supposée violente de son père, ce que rectifie le translateur en ajoutant une promesse de mariage, sans qu'il soit même question encore de grossesse. Il précise ensuite leurs émotions avant leur séparation (Desquelles parolles fut ladicte Bellicenne de son dueil reto[u] rnee en grant lyesse. Et lors prindrent congié l'ung de l'autre, et ne fut pas sans que les yeulx ne fussent contraincts getter babondamment larmes, 11/19-21). Enfin, plus tard, alors que, dans AL, Bellicenne se contentait d'annoncer dans sa lettre la naissance de Baudouin, dans $P R$, elle lui demande en outre de venir la rejoindre (74/5-6). De même, dans la scène d'amour entre Ogier et la reine de Pavie, le prosateur fournit des détails sur leurs ébats qui rendent sa version moins pudique que celle de son prédécesseur, et la scène galante entre Gautier et Clarice reçoit elle aussi un développement patent. Les ajouts apportés à ces trois scènes d'amour témoignent donc d'une influence romanesque plus marquée (et sans doute plus assumée) que dans $A L$.

PR témoigne également du goût de son auteur pour les discours. De nombreux dialogues y sont en effet ajoutés. L'épisode inaugural de la guerre opposant Charlemagne et Geoffroy de Danemark est à ce titre emblématique et programmatique: le récit de la guerre est réduit à une demi-ligne, mais, en contrepartie, est inventé 
un ambassadeur, qui va donner lieu à une surenchère de paroles. Ainsi, d'emblée, la parole se substitue à l'action. Un autre dialogue ajouté dans la quatrième partie corrobore ce premier constat: alors que le poète se contentait d'évoquer la sortie des chrétiens contre les païens qui ont envahi Babylone, le translateur imagine un désaccord entre Gautier et son beau-père Moÿsant et invente une longue scène dialoguée où les chrétiens débattent sur la stratégie à adopter pour faire face à leurs ennemis (\$456-459); en revanche, le récit de la bataille qui suit est vite expédié. Ainsi, là encore, $P R$ privilégie le logos aux dépens de l'action. $P R$ a par ailleurs développé de nombreuses répliques présentes dans $A L$. Remarquable est, par exemple, l'accroissement que subit le récit qu'Ogier fait de ses aventures passées à Caraheu: il passe de 7 vers à presque 40 lignes. Mais l'exemple le plus flagrant de développement des passages dialogués reste celui déjà évoqué par E. Poulain-Gautret, à savoir l'épisode de la cinquième partie relatant l'ensemble des discussions sur la question du remariage de la reine de France et d'Ogier, «logorrhée [ ] peu adaptée à la chanson de geste» ${ }^{27}$. La réécriture est donc marquée par la faveur accordée aux paroles, au détriment bien souvent de l'action.

Le translateur a en particulier amplifié les paroles liées à la religion. Plusieurs prières ont été développées, parfois de façon considérable; cinq prières ont été ajoutées. Le discours apologétique prend aussi plus de poids; $P R$ manifeste même une nette tendance au catéchisme. Ainsi, non seulement les deux leçons dispensées par Naimes à Caraheu sur les icônes et les saints, et celle donnée par Ogier à Moÿsant sur les trois ordres que sont la prêtrise, le mariage et la chevalerie, y conservent leur ampleur, mais le translateur en ajoute encore trois: la première traite de la Trinité, de la Création, de la Damnation et de la Grâce $(\$ 354)$; la deuxième, prise en charge par Guyon, a pour sujets la résurrection du Christ et le Jugement Dernier (413/15-25) la troisième est donnée par Ogier au roi païen Florion, afin de le convertir avant d'entreprendre le duel contre lui (500/14-18). Le discours religieux voit donc sa place nettement augmentée dans $P R$.

L'inflation du discours apologétique s'accompagne d'un développement du discours moralisateur dans $P R$. Cela est patent dans le passage mettant en scène le retour du prince païen Dannemont au palais de Rome, après la déconfiture de son armée: nous y trouvons des leçons de morale prises en charge par son père et sa mère, qui sont absentes du modèle $(\mathbb{2} 23)$. Les répliques que la reine de France adresse à la vieille et fourbe dame de Senlis dans la cinquième partie $(\$ 484,485,488)$, inventées elles aussi, sont un autre exemple flagrant de cette amplification du discours moralisateur dans $P R$.

La comparaison des deux versions met par ailleurs en évidence le développement, parfois considérable, de certaines scènes dans $P R$. Dans la partie consacrée aux Enfances, nous en avons relevées deux en particulier. La première raconte le retour à Suze de l'armée française après sa victoire sur les païens; elle relate la rencontre du pape et la journée passée dans la ville. $A L$ n'y consacrait que 3 vers; $P R$ lui réserve désormais tout un paragraphe qui constitue à lui seul un chapitre. Un tel développement montre de toute évidence que le translateur a voulu privilégier cette victoire

(27) Ibid., p. 110.

(28) Elle s'ajoute aux deux autres leçons de catéchisme qu'a déjà reçues Caraheu dans le cadre de sa conversion. 
de la chrétienté. La seconde scène ayant subi une amplification importante dans les Enfances concerne le rétablissement du pape à Rome. Dans $A L$, le passage était traité en 2 vers; $P R$ la développe sur presque la totalité d'un paragraphe. De telles amplifications témoignent une nouvelle fois du goût du translateur pour la thématique religieuse.

Enfin, $P R$ a inventé plusieurs scènes. Il y en a deux dans la partie consacrée aux Enfances. La première concerne l'épisode du chevage: là où, en effet, le poète se contentait de passer directement de la dénonciation du duc Gauffroy par le traître Hardré au résumé de l'expédition lancée par Charlemagne au Danemark et à l'accord de paix finalement établi, le translateur invente le personnage du premier ambassadeur envoyé par l'empereur auprès du père d'Ogier, ce qui donne lieu, comme nous l'avons déjà signalé, à une nouvelle scène dialoguée qui, malgré ses lourdeurs, a le mérite de fournir une justification vraisemblable au conflit ${ }^{29}$. La seconde addition constitue le paragraphe 20 où l'on voit les douze pairs avertir Charlemagne qu'Ogier a laissé partir indemne un païen, puis le roi convoquer le héros qui lui explique l'accord qu'il a établi avec le païen Sadone. Parmi les autres scènes ajoutées dans $P R$, nous en trouvons en particulier quatre, toutes situées dans les parties nouvelles de la légende, qui mettent en scène l'un des chevaux d'Ogier. Il s'agit tout d'abord de la scène qui évoque le combat des destriers du héros et de Justamon durant leur propre duel. Apparaissent ensuite, dans la dernière partie, trois nouvelles scènes faisant intervenir Papillon, le cheval faé d'Ogier: celle où il tue le potier; celle où il manque de renverser son maître en poursuivant des assaillants; et celle enfin où il étrangle Blanchart, le destrier du roi Philippe. Comme le suggère E. Poulain-Gautret à propos de ces créations, «peut-être s'agit-il de rendre à Papillon les caractéristiques de la race des lutins (par tradition dangereusement facétieux dans les contes), à laquelle il appartient, ou de le charger de la mauvaise part éliminée du personnage de Morgue, trop christianisés ${ }^{30}$.

La réécriture en prose se traduit donc par des réductions et des ajouts qui sont significatifs des nouvelles orientations de son auteur. Mais ces écarts par rapport au modèle témoignent-ils d'un renouvellement, voire d'un «gauchissement» de la matière épique au profit du romanesque, ou bien $P R$ s'inscrit-il toujours dans la continuité de son modèle?

\section{De nouvelles inflexions thématiques?}

Nous nous sommes attachée à l'étude de quatre thématiques - les faits d'armes, les figures du pouvoir royal, le merveilleux et le héros - afin de voir si la réécriture en prose leur réserve encore un traitement épique.

\section{Les faits d'armes}

La thématique des faits d'armes reste omniprésente dans PR. Son auteur a, en effet, conservé toutes les scènes guerrières présentes dans son modèle, même s'il en a abrégé le récit. Leur inventaire révèle aussi la variété et la richesse des situations guer-

(29) Voir M. Отт, Gaufroi de Danemarche et le 'chevage', des décasyllabes aux proses, «Le Moyen Français» 72, 2013, p. 102.

(30) E. Poulain-Gautret, La tradition littéraire d'Ogier le Danois après le XII siècle cit., p. 244. 
rières mises en scène: nous avons toujours, comme dans $A L$, des guerres opposant les chrétiens aux païens (invasions et croisades), des guerres entre chrétiens (conflits entre un suzerain et ses vassaux), des guerres entre Sarrasins (litiges d'ordre privé) auxquels s'ajoutent les combats contre les traîtres, représentés dans les deux camps. Seul le thème de la guerre entre patries chrétiennes n'a pas été exploré. Notons enfin que les épisodes romanesques, notamment amoureux, s'ils fonctionnent comme de courtes pauses dans ce récit à dominante guerrière, sont toujours le théâtre d'affrontements. Dans $P R$, l'épique a ainsi toujours le fin mot sur le romanesque. Quant aux buts poursuivis au travers de ces faits d'armes, ils restent les mêmes que dans $A L$ : convertir l'autre, acquérir les biens d'autrui et se venger. $P R$ n'est donc pas novatrice sur la thématique guerrière.

\section{Les figures du pouvoir royal}

Le translateur n'a pas non plus apporté beaucoup d'innovation dans son traitement des figures du pouvoir royal. Il n'a généralement fait qu'amplifier la démarche déjà à l'œuvre dans les versions antérieures de la légende. Ainsi, la fragilisation du personnage de Charlemagne, déjà présente dans La Chevalerie Ogier et renforcée dans $A L$, s'accentue encore dans $P R$. Le translateur développe en effet des scènes traduisant sa propension à la colère associée à des intérêts personnels, ses faiblesses à l'égard de son fils et de l'argent, son impuissance à régler des conflits; il procède également à une distanciation entre le roi et Dieu, en supprimant notamment les visites d'anges venus réconforter Charlemagne. Tout cela contribue à désacraliser davantage le personnage, qui fonctionne souvent comme un contrepoint du héros.

La dégradation de la figure royale passe toujours aussi dans $P R$ par le personnage de Charlot. Générateur de troubles, il reste porteur d'une bonne part de l'image négative du pouvoir. Soulignons toutefois que le translateur fait disparaitre le passage, compromettant pour le prince, qui mentionnait sa fuite avec les traîtres, son court séjour hors de France et sa mort à son retour. L'auteur de $P R$ a également retouché l'épisode en diminuant la part de responsabilité de Charlot: désormais, c'est le duc de Normandie qui persuade avec habilité le prince de participer à un complot contre Gautier, et Rohard, le champion des traitres, une fois vaincu, le décharge de toute responsabilité, remettant toute la faute sur le duc de Normandie. Le prosateur fait même ajouter à Gautier: car je sçay bien que il n'a pas trouvé ne cerché la trabyson qui m'a esté mise sus (370/22-23). Ces modifications pourraient être le signe d'«un mouvement de respect», comme le suggère E. Poulain-Gautret ${ }^{31}$, mais elles nous semblent aussi traduire la volonté de l'auteur de $P R$ de ne pas rendre trop caricatural son personnage et d'en laisser un portrait nuancé. Le personnage de Charlot, par son ambiguïté, et par conséquent sa richesse sur le plan narratif, est éminemment romanesque. Comme son père, mais de façon plus accentuée, il contribue à affaiblir et ternir la figure royale; il met aussi en question le principe intangible de la transmission familiale du pouvoir royal. Son personnage offre enfin un contrepoint du héros et de son double mineur, Gautier.

L'autre action du prosateur sur les figures du pouvoir concerne le roi Philippe, dont la fragilisation est encore augmentée à travers quelques ajouts et amplifications significatifs. Il devient notamment l'objet de railleries de la part de ses vassaux, et son évincement au profit de son épouse y est nettement accentué.

En somme, toutes les figures royales de $P R$ sont fragilisées. Notons juste qu'en contrepartie, $P R$ insère deux passages louant l'attribution du pouvoir par élection: le 
discours de Godebeuf sur le pouvoir à Acre (302/7-10), et celui de l'épouse du roi Philippe (522/15-20).

\section{Le merveilleux}

Le miraculeux chrétien a encore sa place dans $P R$, mais il n'est l'objet d'aucune invention et demeure stéréotypé. Nous pouvons sans doute y voir le signe du vieillissement de l'inspiration liée au miraculeux épique au profit du merveilleux féérique. Quant au merveilleux féérique et breton, s'il est plus productif et donne lieu à certaines trouvailles, il a néanmoins subi des coupes. Le translateur a en fait opéré un rééquilibrage entre les deux formes de merveilleux, en faisant évoluer le rapport qu'ils entretiennent entre eux, mais aussi leurs rapports avec la matière épique: d'une part, toute tension entre le merveilleux chrétien et le merveilleux féérique a disparu, le second étant désormais au service de l'autre (à travers la christianisation de Morgue); d'autre part, le merveilleux féérique ne s'oppose plus non plus à la matière épique: il est un expédient pour préserver le héros épique et le rendre capable d'accomplir de nouveaux exploits pour protéger la chrétienté. De fait, il permet à Ogier de continuer d'être un héros épique.

\section{Le héros}

Ogier est toujours dans $P R$ un être hors norme, un être d'exception, et en cela il reste un héros. C'est également un personnage voué à être hors du groupe, en marge de la communauté, et $P R$ offre ainsi toujours une vision tragique du héros épique. Il est toujours aussi un héros au centre du récit et du discours des autres personnages.

Pourtant des signes de fragilisation plus marqués apparaissent dans la dernière partie, à travers notamment la présence de cinq scènes parodiques particulièrement développées, dont deux sont de pures inventions du translateur. Le passage d'Ogier en Faerie a en effet altéré son héroïsme avec notamment l'acquisition d'adjuvants merveilleux, mais l'a-t-il pour autant fragilisé? La quatrième partie amorce-t-elle un mouvement de gauchissement de l'héroïsme épique dans la mesure où la nouvelle nature merveilleuse d'Ogier donne désormais lieu à des scènes parodiques? D'après nous, s'il y a effectivement «vacillement» ${ }^{32}$ du héros épique dans $P R$, nous n'assistons pas pour autant à sa destruction: ces scènes ne sont pas en effet accompagnées de commentaires dépréciatifs de la part du narrateur; de plus elles sont circonscrites, la parodie étant exclue du restant du récit de cette dernière partie; enfin, leur impact est limité et le problème occasionné est vite résolu et n'a pas de conséquence pour la suite, excepté dans le cas de la première scène de la perte de l'anneau, mais la trahison de la dame de Senlis se résout par un combat dont sort victorieux Ogier. Mieux, au terme des mésaventures liées à la perte de l'anneau, le héros bénéficie d'une reconnaissance, voire d'une glorification. Nous interprétons finalement ces scènes de reconnaissance comme des modèles de lecture invitant à considérer les épisodes parodiques comme des intermèdes plaisants, comiques, qui n'enlèvent rien à l'héroïsme d'Ogier. L'impact de ces scènes sur le héros serait moins alors à interpréter en termes de dégradation que d'altération. Elles ne font qu'offrir une nouvelle image, burlesque et comique, du héros, sans qu'il perde pour autant sa nature épique qui lui

(32) E. Hoyer-Poulain, Ridicule d'Ogier: vacillements du béros dans les remaniements de la Chanson d' "Ogier le Danois", dans Burlesque et dérision dans les épopées de l'Occident médiéval, Besançon, Annales littéraires de l'Université de Besançon, 1995, pp. 49-57. 
est intrinsèque; mieux, ces nouveaux traits ne seraient là que «pour mieux préserver ailleurs son personnage épique» ${ }^{33}$.

En conclusion, $P R$ n'est pas un dérimage, mais une véritable réécriture s'inscrivant à la fois dans la continuité et le renouvellement de son modèle. Le translateur a su créer une nouvelle forme qui fait sens, au carrefour des traditions épiques et romanesques. L'édition de la version française de la prose la plus ancienne d'Ogier le Danois s $^{34}$ présente alors plusieurs intérêts. Elle donne d'abord accès à une œuvre de qualité, à la composition cohérente et significative. De plus, si elle ne présente pas d'intérêt historique particulier, elle offre néanmoins un nouveau champ d'investigation en matière d'histoire et de théorie littéraires: elle permet d'appréhender les problèmes posés par l'adaptation de la poésie épique et nous éclaire sur la postérité des chansons de geste. Elle est de fait une nouvelle pierre à l'édifice des recherches sur les mises en prose, trop longtemps dépréciées par les médiévistes au profit des chansons de geste versifiées.

AURÉLIA DOMPIERRE

(33) E. Poulain-Gautret, La Tradition littéraire d'Ogier le Danois après le XIII siècle cit., p. 342.

(34) Nous en préparons actuellement la publication. 